\title{
The ACS LCID Project: Quantifying the Delayed Star Formation in Leo A
}

\author{
Andrew A. Cole ${ }^{1}$ \\ for the LCID Team $\dagger$ \\ ${ }^{1}$ Department of Astronomy, University of Minnesota, 116 Church St. SE, \\ Minneapolis, MN, 55455, USA \\ email: cole@astro.umn.edu
}

\begin{abstract}
We have obtained deep images of the Local Group dwarf irregular galaxy Leo A using the Hubble Space Telescope Advanced Camera for Surveys. From these images we have constructed the deepest ever color-magnitude diagram (CMD) for an irregular galaxy beyond the Magellanic Clouds. We derive the star-formation rate (SFR) as a function of time over the entire history of the galaxy. We find that over $90 \%$ of Leo A's star formation has occurred more recently than 8 Gyr ago. Our measurements are not inconsistent with a scenario in which all of the ancient star formation occurred prior to the end of the era of reionization, with subsequent suppression until lookback times corresponding to redshift $z \approx 1$. However, given Leo A's strongly isolated state and its dynamical mass of $\gtrsim 10^{8} \mathrm{M}_{\odot}$, its large gas supply was more likely to have been merely heated than driven out of the galaxy altogether.
\end{abstract}

Keywords. galaxies: dwarf, color-magnitude diagrams, galaxies: evolution

\section{Introduction}

Dwarf galaxies are the most common class of galaxy in the Universe, which gives them significance far beyond their mass. They are simpler in structure and perhaps more easily understood than giant galaxies, and so their study can shed light on the processes governing galaxy evolution. Because the relative importance of mergers and interactions as compared to intrinsic properties is not well-known, it is important to study dwarfs in as many environments as possible. Therefore we have begun a Hubble Space Telescope Advanced Camera for Surveys (ACS) program to measure the complete star-formation histories $(\mathrm{SFH})$ of six isolated Local Group dwarf galaxies in order to search for the imprints of cosmological processes on their evolution (Gallart et al., in preparation). By measuring several small, isolated galaxies, we will test for correlations and patterns in their SFH that may provide clues as to the magnitude of the effects of reionization, supernova blowout, and other feedback processes.

Here we present our first results, a new measurement of the SFH of the galaxy Leo A (DDO 69). Leo A was discovered by Zwicky (1942) in the course of a search for the lowest luminosity galaxies. Leo $\mathrm{A}$ is a small, blue galaxy conspicuous for its population of young, massive stars, with estimated ages from $\approx 10^{7}-10^{8} \mathrm{yr}$ (Tolstoy et al. 1996). The star-formation rate (SFR) has apparently declined since that time, although a few small $\mathrm{H}$ II regions are present. It is $800 \mathrm{kpc}$ from the Milky Way, and over $1000 \mathrm{kpc}$ from M31, making it one of the most isolated galaxies in the Local Group.

The first HST studies of Leo A (Tolstoy et al. 1998; Schulte-Ladbeck et al. 2002) found strong evidence for a high fraction of young stars in a centrally concentrated distribution, but failed to reach the necessary depth to convincingly measure the fraction

$\dagger$ LCID: Local Cosmology from Isolated Dwarfs 


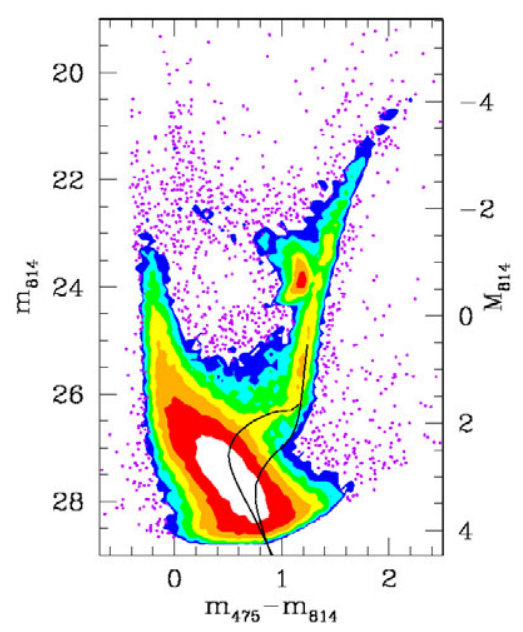

Figure 1. Our ACS/WFC CMD for Leo A. Individual stars are plotted where their density is less than 8 stars $/$ decimag $^{2}$. Contours are uniformly spaced by factors of two to show the overall density distribution and the fine structure of the stellar sequences. The black lines show the main-sequence and lower RGB of isochrones with $(\mathrm{Z}$, age/Gyr $)=(0.0001,14)$ and $(0.001,5)$, respectively.

and age distribution of the oldest stars. Dolphin et al. (2002) supplied the first proof of the existence of truly ancient stars in Leo A with the discovery of RR Lyrae type variables.

\section{Data Acquisition and Photometry}

Leo A was observed with the HST/ACS for a total of 16 orbits, split evenly between the F475W (Sloan $g^{\prime}$ ) and F814W (Broad I) filters. The Holmberg major axis of Leo A is $3.2^{\prime}$, and the total extent of the galaxy as traced by neutral hydrogen and by red giant stars is about $15^{\prime}$. Our field is located almost entirely within the central part of the galaxy.

We obtained photometry for $\approx 112,000$ stars using the DOLPHOT point spread function-fitting software (Dolphin 2000). Simulations show that the typical photometric error reaches $\pm 0.1 \mathrm{mag}$ for $\left(\mathrm{m}_{475}, \mathrm{~m}_{814}\right)=(28.7,27.9)$; the $50 \%$ completeness limit is at $\left(\mathrm{m}_{475}, \mathrm{~m}_{814}\right)=(29.0,27.9)$. The CMD is shown in Figure 1. Where the density of stars is high enough to confound the easy estimation of the relative number of stars in the various sequences, we have plotted contours corresponding to stellar density rather than individual stars. The contours cover the range from 8-512 stars/decimag ${ }^{2}$, evenly spaced by factors of 2 .

The main features which bear on the measurement of the SFH, especially at the previously ill-constrained earliest times, are the relatively bright locations of the peak density of subgiants and the main-sequence turnoff; the underdeveloped horizontal branch and strong, vertically extended red clump; and the paucity of upper red giant branch stars. The implication of the bright subgiant branch is immediately evident by comparison to the location of the old, metal-poor isochrone in Fig. 1: 14 Gyr old stars would produce a far fainter subgiant branch than observed. Because our photometry reaches the oldest main-sequence turnoff, we can unambiguously quantify the galaxy's youth for the first time. 


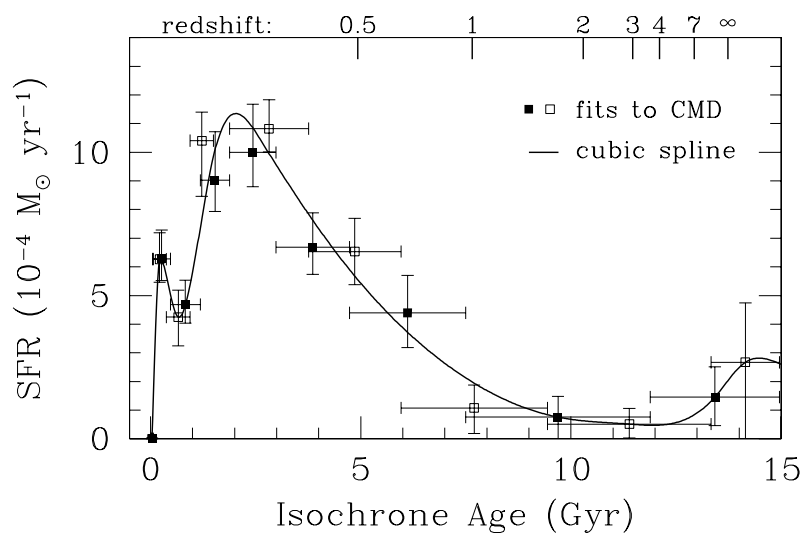

Figure 2. The derived SFH of central Leo A. Data points with errorbars show the best fits to the CMD for two age binning schemes. The heavy line shows the results of a spline fit to the results. The equivalent redshift based on third-year WMAP results and flat $\Lambda$ CDM cosmology is given along the top axis.

\section{Derivation of the Star-Formation History}

We derive the SFH of Leo A by statistical comparison of the CMD to the stellar distributions predicted by a set of theoretical models, convolved with the appropriate observational biases and Poisson errors. We use isochrones from the Padua group (Girardi et al. 2000) in our derivation, creating a large number of synthetic CMDs and searching for the combination of parameters that best reproduces the data. Extensive tests of the technique have shown that it is insensitive to the details of the fitting procedure and that the shape of the derived $\mathrm{SFH}$ is robust to any reasonable change in parameters (e.g., distance, reddening, IMF) or choice of isochrones. We adopt $(\mathrm{m}-\mathrm{M})_{0}=24.5$ and $\mathrm{E}(\mathrm{B}-\mathrm{V})=0.02$ in our calculations (Dolphin et al. 2002).

We solved for SFR as a function of age in 9 logarithmically spaced bins. Within each age bin, a range of metallicities was allowed, from $0.0001 \leqslant \mathrm{Z} \leqslant 0.0015$. The only constraint on age-metallicity relation was to exclude the lowest metallicity isochrones from the youngest age bins, as they are seen by inspection to be mismatched to the data. Our derived history of star formation in Leo A is shown in Figure 2. The best-fit SFH is shown for two different age binnings- solid squares and open squares, respectively. Each point carries its $1 \sigma$ errorbar in SFR and an age errorbar marking the width of the age bin. The heavy curve is a cubic spline fit to the results; it represents our best estimate of the form of the SFH in the central part of Leo A. The redshift scale at top applies to a flat $\Lambda \mathrm{CDM}$ universe with parameters taken from the WMAP year-3 dataset (Spergel et al. 2006).

Because of age-metallicity degeneracy, a shift in the peak SFR to ages possibly as high as 4-5 Gyr is not strongly ruled out by these data. The derived rates are normalized to the area of the field of view, $0.6 \mathrm{kpc}^{2}$. Leo A is best described as a late-blooming galaxy, in which the great majority of stars are formed after a delay of several Gyr. The apparent early peak of star formation above a subsequent minimum is not a highconfidence feature, as the errorbars indicate. We omit the derived metallicity evolution because there is large scatter around a nearly flat age-metallicity relation, with almost no trend: constant $\mathrm{Z}=0.0008_{-0.0003}^{+0.0005}$ is an adequate representation of the results, although there is some hint of a trend of increasing metallicity with time, from $\mathrm{Z}=0.0006$ to $\mathrm{Z}=$ 0.001 . 


\section{Summary \& Discussion}

Within the ACS/WFC field of view, $90 \%$ of the star formation has occurred more recently than 8 Gyr ago. However, some truly ancient stars are required to reproduce the CMD and provide the observed RR Lyraes. The 14 Gyr isochrone in Fig. 1 traces the lower envelope of subgiant stars, confirming that not all of the star formation was delayed.

By integrating over a radial profile (Vansevičius et al. 2004), we find that Leo A only astrated $\sim 1-2 \times 10^{6} \mathrm{M}_{\odot}$ at ages corresponding to redshifts $z>1$. If all of its presentday baryon inventory was present at high redshift, then Leo A would have been $95 \%$ gas and only $5 \%$ stars prior to $z \approx 2$. Evidently some small galaxies can retain or build large gas reservoirs without much depletion by star formation or loss to the intergalactic medium. This makes Leo A-like objects intriguing candidates for proto-galactic fragments that merge into giant galaxies, prompting bursts of star formation without leaving much stellar residue in their halos.

Leo A is not the only "late bloomer" in the Local Group, but it appears to be an extreme case. An example of a qualitatively similar SFH can be found in the Leo I Gallart et al. (1999) dwarf spheroidal (dSph) galaxy. The two galaxies are of comparable mass, but the latter is a gas-free, bound satellite of the Milky Way, with plausible triggers for late star formation in tidal interactions or accretion events. A trigger in the case of Leo $\mathrm{A}$ is harder to identify.

Given its probable long history of isolation, it may be that much of the gas in Leo A was present from early on, and therefore the suppression of early star formation is not attributable to the loss or exhaustion of its initial reservoir. It seems more plausible that only a small fraction of the $\mathrm{H}$ I was able to participate in star formation, with the rest kept warm in a halo; the UV background or supernova feedback are candidate heat sources. Sequestering most of the gas in a halo also speeds the chemical evolution clock, which is interesting given the prompt enrichment and subsequent flat age-metallicity relation in the galaxy. The warm halo would have been diffuse and metal-poor, resulting in a long cooling timescale and a possible delay before it could participate in star formationperhaps triggered by a rare infall or interaction event in this isolated dwarf.

\section{Acknowledgements}

Support for this work was provided by NASA through grant GO-10590 from the Space Telescope Science Institute, which is operated by AURA, Inc., under NASA contract NAS5-26555.

\section{References}

Dolphin, A.E. 2000, PASP, 112, 1383

Dolphin, A.E., Saha, A., Claver, J., Skillman, E.D., Cole, A.A., Gallagher, J.S., Tolstoy, E., Dohm-Palmer, R.C., \& Mateo, M. 2002, AJ, 123, 3154

Gallart, C., Freedman, W.L., Aparicio, A., Bertelli, G., \& Chiosi, C. 1999, AJ, 118, 2245

Girardi, L., Bressan, A., Bertelli, G., \& Chiosi, C. 2000, A\&AS, 141, 371

Schulte-Ladbeck, R.E., Hopp, U., Drozdovsky, I.O., Greggio, L., \& Crone, M.M. 2002, AJ, 124, 896

Spergel, D.N., et al. 2006, ApJ, submitted, astro-ph/0603449

Tolstoy, E. 1996, ApJ, 462, 684

Tolstoy, E., Gallagher, J.S., Cole, A.A., Hoessel, J.G., Saha, A., Dohm-Palmer, R., Skillman, E., Mateo M., \& Hurley-Keller, D. 1998, AJ, 116, 1244

Vansevičius, V., et al. 2004, ApJ, 611, L93

Zwicky, F. 1942, Phys. Rev., 61, 489 


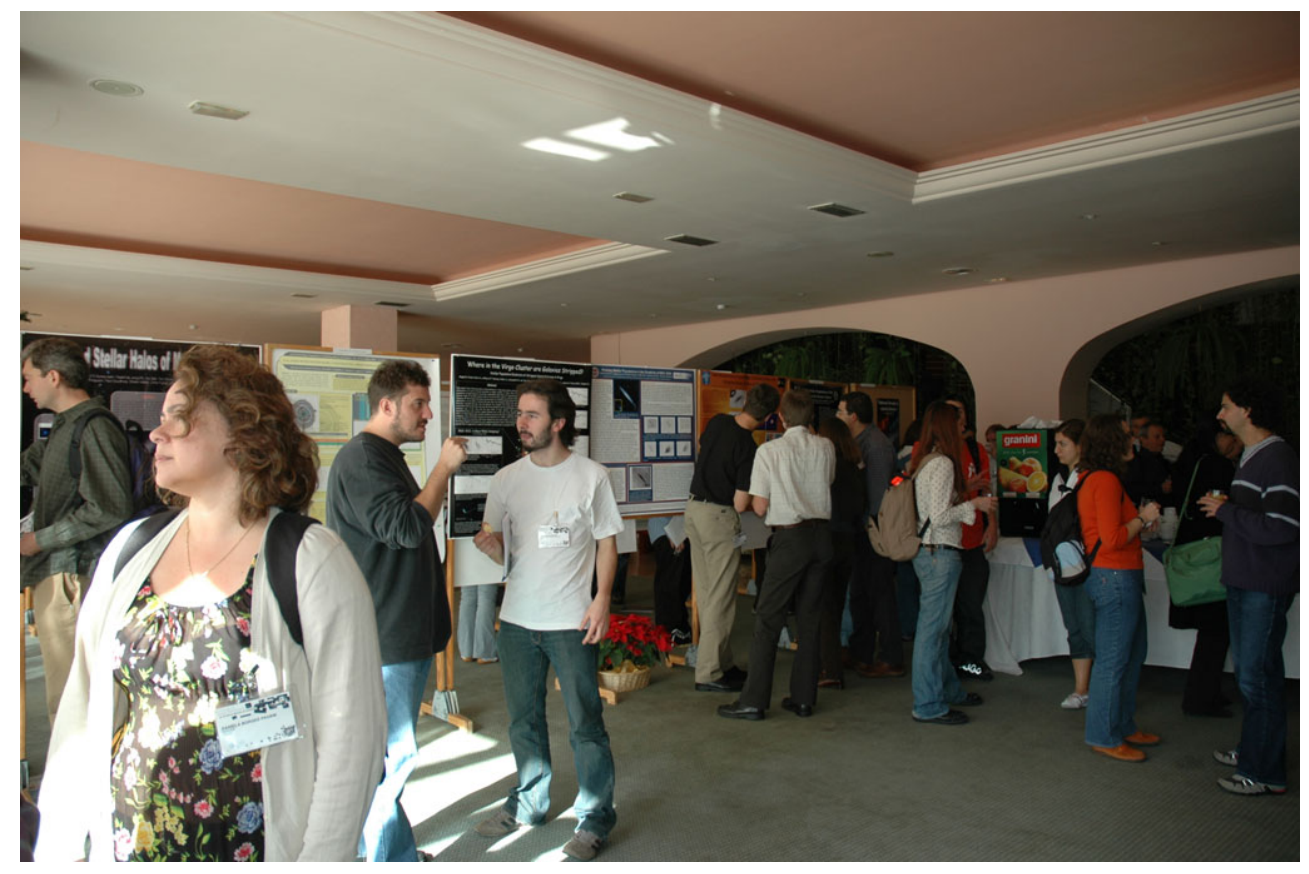

The poster area. 